Research Article

\title{
Study of the Windowing and Overlap-Add Operation for a Super-Gaussian Random Vibration Test
}

\author{
Yiming Peng $\mathbb{D}^{1}$ and Yang $\mathrm{Li} \mathbb{D}^{2}$ \\ ${ }^{1}$ State Key Laboratory of Mechanics and Control of Mechanical Structures, Nanjing University of Aeronautics and Astronautics, \\ Nanjing 210016, China \\ ${ }^{2}$ School of Aeronautic Engineering, Nanjing Vocational University of Industry Technology, Nanjing 210023, China
}

Correspondence should be addressed to Yang Li; 2018100940@niit.edu.cn

Received 19 November 2020; Revised 17 April 2021; Accepted 14 May 2021; Published 29 May 2021

Academic Editor: Carlo Rainieri

Copyright $\odot 2021$ Yiming Peng and Yang Li. This is an open access article distributed under the Creative Commons Attribution License, which permits unrestricted use, distribution, and reproduction in any medium, provided the original work is properly cited.

\begin{abstract}
Random vibration environmental testing employs the specified statistical properties of the real world vibration to reproduce the desired excitations on the shaker table for fatigue test purposes. Smooth and safe operation is the essential requirement for a longduration test. Traditionally, the windowing and overlap-add (WOA) method is applied to the acceleration signals of the shaker table, and previous studies have indicated that this operation reduces the kurtoses of the processed signals. To protect the test equipment from abrupt changes in the input voltage, the WOA method is proposed to operate on the input voltage signals in a frame-by-frame form for super-Gaussian environmental testing. To figure out the impacts of the proposed operation on the response kurtoses of a shaker table, we express the system transfer function in the time domain, and the WOA method is analysed considering the transfer function of a dynamic system. Based on the analysis, a further study is made to explain the mechanism of the kurtosis decrease due to the WOA method. Through the study, we find that the kurtosis reduction conclusion is not applicable to all types of super-Gaussian signals, and the kurtoses can be invariable and even increased by allocating the positions of the highexcursion peaks of super-Gaussian signals when the WOA method is applied. A window function is recommended for zeromemory nonlinear (ZMNL) transformation to move the positions of the high-excursion peaks of a super-Gaussian signal, providing a novel way of adjusting kurtosis when WOA method is applied. The proposed WOA method and window function are first verified in a single-input-single-output (SISO) numerical simulation to test their effectiveness under different reference kurtoses. Then, they are evaluated in a two-input-two-output shaker table test. The test results demonstrate that the proposed window function can prevent the kurtosis decrease with the application of the WOA method.
\end{abstract}

\section{Introduction}

The windowing operation is widely used in the signal processing domain. For example, in spectral analysis, computers are not capable of handling signals with infinite length. A common practice is to split the signals into segments on which Fourier transformation is applied. However, the truncation may result in a distortion of the frequency spectra of the original signals [1]. To mitigate this issue, the intercepted signals are multiplied by window functions. Another operation, known as the overlap-add (OLA) method, reassembles the different pieces of processed signals into infinite-length signals. Usually, the OLA method is often applied after the windowing operation.

The windowing and overlap-add (WOA) operation is also an important tool in random vibration environmental testing. In the test, products are exposed to the vibrational environments they endure during their service lifetime. The vibration environments we apply in the laboratory should possess the same statistical properties as those in the real world. These properties include the power spectra density (PSD), probability density function (PDF), kurtosis and skewness for the non-Gaussian random test, and stationarity for the stationary random test. These objects frequently 
deviate from their desired values due to disturbances and some indispensable algorithms. The WOA method is the dispensable method in this test. The vibration test serves for testing the reliability or fatigue assessment, which will take a considerably long time. The shaker table excitations with long-enough duration are required. However, the memory of most test instruments is unable to save and process the entire signals with the desired length. As a result, the excitations are generated segment by segment. Each segment should possess the desired statistical properties. WOA method is used to connect two segments in case discontinuity between the two segments of signals occurs. In this area, the major concern lies in the impact of the WOA method on the above statistical properties. Many research works have been conducted to mitigate the side effects. In 1993, time-domain randomization was recommended for the Gaussian stationary random test by Smallwood and Paez [2]. The method can produce infinite-length input signals without changing the PSD of the output signals. In addition, several window functions and overlap factors were studied for the realization of the minimum stationarity errors. In 1997, Smallwood [3] put forward a procedure for super-Gaussian tests. In that article, he indicated that the generation of continuous and infinite inputs by the means of time-domain randomization presents some difficulties. If the WOA operation, the core component of time-domain randomization, is implemented in a super-Gaussian test, the output kurtoses are reduced, and the distribution of the output moves towards a Gaussian distribution. In 2005, Steinwolf et al. [4] investigated the kurtosis reduction arising from the WOA method for fatigue estimation and put forward a equation to describe the numerical relationship of the reduction. They located the specific numerical relationship between the kurtosis of the signal before WOA operation and the signal's kurtosis after the operation. In 2019, González and López-Valcarce [5] put forward a window design method to improve the stationarity for both the Gaussian and non-Gaussian random tests. They reported that the OLA method shifted the random process from super-Gaussian to Gaussian. In general, the current viewpoint is that the WOA operation reduces the kurtosis of the super-Gaussian signal to be processed. Currently, there are only two methods to solve the problem. One is to simply employ the kurtosis control algorithm [6]. The other is to use Steinwolf's equation [4]. The equation is not always precise, and additional kurtosis control efforts are still needed after the correction. How to eliminate the kurtosis loss effectively and accurately is the concern of this article.

After 2010, fatigue studies for structures under superGaussian random excitations are widely conducted. A series of articles has been published in the area [7-10]. In fatigue estimation, the acceleration signals of the shaker table are seen as the ground excitations of structures. To maintain the continuity of the long-duration excitations, the WOA method is applied to the acceleration signals. This type of operation can be found in many literature reports [11-14]. The foremost input of the whole test system is the input voltage of the shakers. We can control the shape of the PSD, kurtosis, and other properties of any location in the system (points on shaker table or on under-test structures) by modifying the input voltage. The continuity of the input voltage is the basic requirement for a smooth and safe experiment. Usually, the loss of WOA operation leads to considerably large peaks in the voltage level at the connecting area of adjacent frames. The consequence of the large voltage difference is the abrupt amplitude change of the shaker table, which may damage the shaker coil and disrupt an ongoing fatigue test. Articles about the WOA operation on input voltage signals have not been reported in the literature.

Motivated by the above considerations, we apply the WOA method to input voltage signals of a shaker table test system. First, the WOA operation and its impact on output kurtoses are studied. Here, the output denotes the acceleration response of a shaker table, which usually acts as excitations of the structures fixed on the table in a fatigue or reliability test. Then, the reason for the kurtosis reduction caused by the WOA operation is researched. Finally, a window function is introduced in the process of generating super-Gaussian signals using a zero-memory nonlinear (ZMNL) transformation. With this windowing operation, the kurtosis reduction can be removed. The article is organized as follows. We introduce the definition of Gaussian and non-Gaussian random processes in Section 2, including their differences in kurtoses, the amplitude of time-domain signals, and PDFs. Then, the state-space method of formulating transfer function matrices of a dynamic system is introduced in Section 3. In Section 4, we introduce the WOA method. Then, the impact of the WOA method on the kurtoses of the shaker table's acceleration signals is deduced. A numerical simulation is provided here to verify this deduction. In Section 5, a windowed ZMNL transformation is proposed. A numerical example is given to verify the benefits of the proposed method. A super-Gaussian field test is given in Section 6 to validate the proposed method in a two-inputtwo-output case. Finally, a discussion is presented and conclusion summarized in Section 7.

\section{Gaussian and Super-Gaussian Random Processes}

The kurtosis of any time-domain signal $x(t)$ is defined as the fourth central moment divided by the square of its second central moment. In particular, when the mean of $x(t)$ is zero, the kurtosis can be expressed as [15]

$$
K=\frac{E\left[x^{4}(t)\right]}{E^{2}\left[x^{2}(t)\right]}
$$

where $\mathrm{E}$ denotes the expected value. The kurtosis of a Gaussian signal is approximately 3. A non-Gaussian signal with a kurtosis greater than 3 is called a leptokurtic or superGaussian signal; it is platykurtic or sub-Gaussian when this value is less than 3 . Intuitively, leptokurtic time histories have much more high-excursion peaks than Gaussian and platykurtic signals, as seen in Figure 1. The time signals pictured in Figure 1 share the same amplitude spectra but have different kurtoses. With a rise in kurtosis, high peaks 

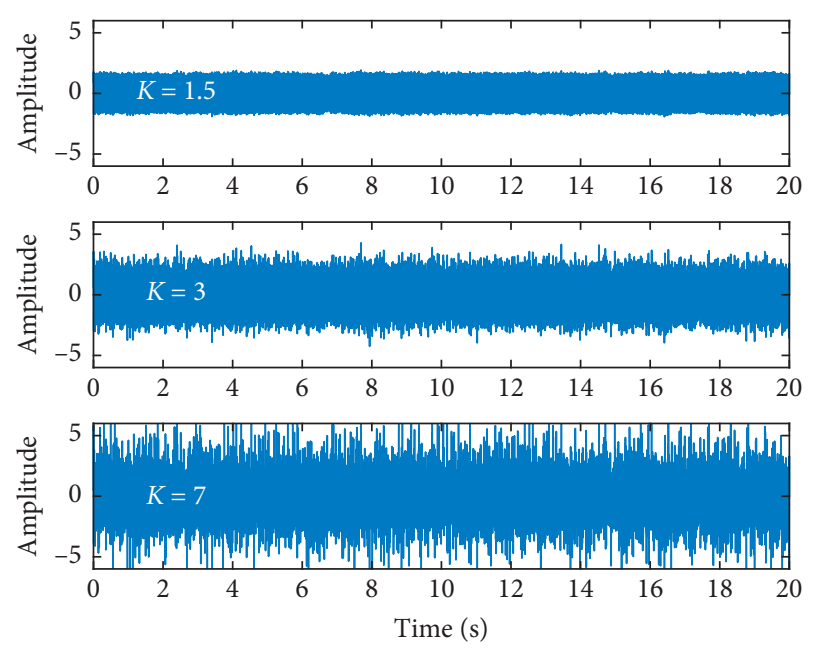

FIgURE 1: Three random time histories with different kurtoses.

increase in quantity and height. This conclusion can also be reached by observing the PDFs of the three signals shown in Figure 2. Actually, the kurtosis characterizes the sharpness of the PDF head and the width of the PDF tails. A superGaussian signal has a sharper PDF head and wider PDF tails than a Gaussian or sub-Gaussian signal does.

\section{State-Space Method}

The time-domain procedure for the super-Gaussian test used in the submission was presented by Cui et al. [16]. The formulation of this procedure starts with expressing the system transfer function using state-space matrices.

The equation of motion of an $\mathrm{N}$ degree-of-freedom system can be written as

$$
M \ddot{x}+C \dot{x}+K x=L_{f} f
$$

where $\ddot{x}, \dot{x}$, and $x$ are the system's response components representing the acceleration, velocity, and displacement, respectively; $M, C$, and $K$ are the mass, damping, and stiffness matrices, respectively. $f$ is the vector of external excitation forces that act on the structures, and $\mathrm{L}_{\mathrm{f}}$ is the mapping matrix for the input forces. The equation of motion shown in (2) can also be expressed in a continuous statespace form as

$$
\dot{z}=A_{c} z+B_{c} f
$$

where

$$
\begin{aligned}
\mathrm{z} & =\left[\begin{array}{c}
\mathrm{x} \\
\dot{\mathrm{x}}
\end{array}\right], \\
\mathrm{A}_{\mathrm{c}} & =\left[\begin{array}{cc}
0 & \mathrm{I} \\
-\mathrm{M}^{-1} \mathrm{~K} & -\mathrm{M}^{-1} \mathrm{C}
\end{array}\right], \\
\mathrm{B}_{\mathrm{c}} & =\left[\begin{array}{c}
0 \\
\mathrm{M}^{-1} \mathrm{~L}_{\mathrm{f}}
\end{array}\right] .
\end{aligned}
$$

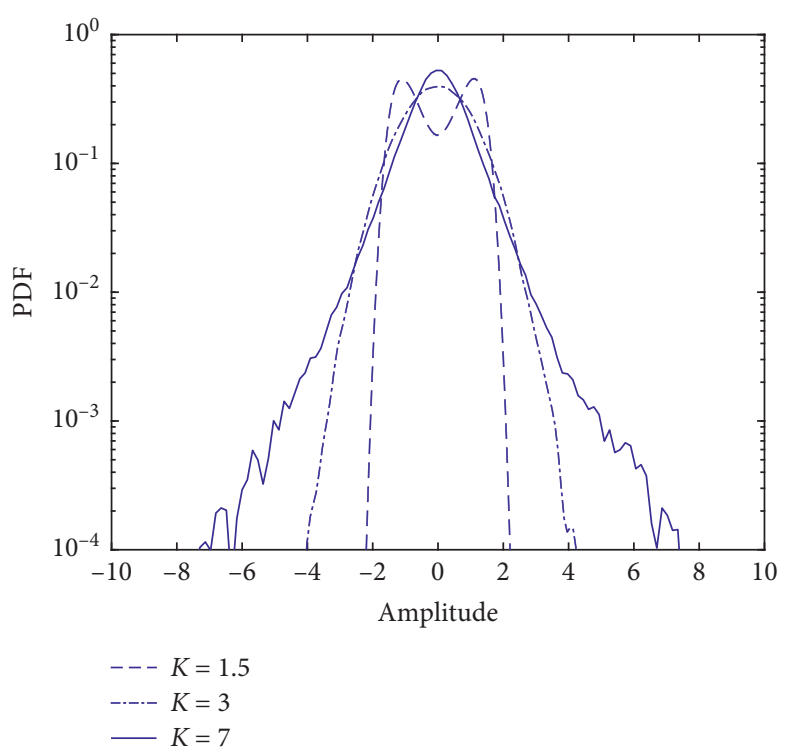

FIgURe 2: Probability distribution of three signals with different kurtoses.

Subscript $c$ denotes matrices for a continuous system. Vector $y$ is the output of the structure and can be assembled from the measurements with

$$
y=R_{a} \ddot{x},
$$

where $R_{a}$ contains the output influence matrices for the measured accelerations. Equation (5) can be rewritten as

$$
y=C_{c} z+D_{c} f
$$

where

$$
\begin{aligned}
& C_{c}=\left[-R_{a} M^{-1} K-R_{a} M^{-1} C\right], \\
& D_{c}=R_{a} M^{-1} L_{f} .
\end{aligned}
$$

In practice, the measurements are recorded over discrete time intervals, and the continuous state-space equation should be transformed into discrete form. Therefore, (3) and (6) can be converted into the following equations:

$$
\begin{array}{r}
z(j+1)=A_{d} z(j)+B_{d} f(j), \\
y(j)=C_{d} z(j)+D_{d} f(j),
\end{array}
$$

where $A_{d}, B_{d}, C_{d}$, and $D_{d}$ are the discrete version of the system state-space matrices. Several sample and hold algorithms $[17,18]$ are useful for solving discrete state-space matrices. The regularly adopted algorithm is the zero-order hold, and the corresponding $A_{d}, B_{d}, C_{d}$, and $D_{d}$ are expressed as

$$
\begin{aligned}
& A_{d}=\exp \left(A_{c} \cdot d t\right), \\
& B_{d}=\left(A_{c}\right)^{-1}\left(A_{d}-I\right) B_{c}, \\
& C_{d}=C_{c}, \\
& D_{d}=D_{c},
\end{aligned}
$$

where $d t$ is the discrete time interval. 
For a known input sequence $f$ and initial state $z(0)$, the state vectors at any time step can be written as

$$
z(j+1)=A_{d}^{j+1} z(0)+\sum_{i=0}^{j} A_{d}^{i} B_{d} f(j-i) .
$$
(10):

Thus, the system output can be derived based on (8) and

$$
y(j)=C_{d} A_{d}^{j} z(0)+D_{d} f(j)+\sum_{i=0}^{j-1} C_{d} A_{d}^{i} B_{d} f(j-1-i) .
$$

For zero initial conditions, i.e., $z(0)=0$, (11) can be represented as a moving average model:

$$
y(j)=\sum_{i=0}^{j} H_{i} f(j-i),
$$

where the values of $H_{i}$ are called the system Markov parameters, which are often used for the time-domain force identification. Equation (12) can also be rewritten in the form of the matrix convolution equation as

$$
y_{0}=H f_{0},
$$

where

$$
\begin{aligned}
& \mathrm{y}_{0}=\left[\begin{array}{c}
\mathrm{y}(0) \\
\mathrm{y}(1) \\
\vdots \\
\mathrm{y}(\mathrm{n}-1)
\end{array}\right], \\
& \mathrm{H}=\left[\begin{array}{cccc}
\mathrm{H}_{0} & 0 & \cdots & 0 \\
\mathrm{H}_{1} & \mathrm{H}_{0} & 0 & \vdots \\
\vdots & \vdots & \ddots & 0 \\
\mathrm{H}_{\mathrm{n}-1} & \mathrm{H}_{\mathrm{n}-2} & \cdots & \mathrm{H}_{0}
\end{array}\right] \text {, } \\
& f_{0}=\left[\begin{array}{c}
f(0) \\
f(1) \\
\vdots \\
f(n-1)
\end{array}\right] \text {. }
\end{aligned}
$$

Here, $\quad H \in \mathbb{R}^{\left(\mathrm{N}_{\mathrm{m}} \cdot \mathrm{n}\right) \times\left(\mathrm{N}_{\mathrm{f}} \cdot \mathrm{n}\right)}, \quad \mathrm{y}_{0} \in \mathbb{R}^{\left(\mathrm{N}_{\mathrm{m}} \cdot \mathrm{n}\right) \times 1}, \quad$ and $\mathrm{f}_{0} \in \mathbb{R}^{\left(\mathrm{N}_{\mathrm{f}} \cdot \mathrm{n}\right) \times 1}$, where $N_{m}$ is the number of sensors; $n$ is the number of sampling points of every block, and $N_{f}$ is the number of excitations. According to (11), the system Markov parameters can be expressed as

$$
H_{i}= \begin{cases}D_{d}, & i=0, \\ C_{d} A_{d}^{i-1} B_{d}, & i=1,2, \ldots, n-1 .\end{cases}
$$

Acquiring system Markov parameters, as shown in (15), the desired time-domain response signals for environmental testing are required so that we can compute the input forces for the under-test dynamical systems. The traditional environmental test procedure is defined in the frequency domain, and there are no such time-domain signals.
Nevertheless, we can construct this kind of signal using reference spectra defined in the frequency-domain test procedure. The Cholesky decomposition of the reference spectra $R$ is

$$
R=L L^{H}
$$

where the superscript $H$ denotes the operation of the conjugate transpose. Multiplying the Cholesky factor $L$ with the phase matrix $P$ as in (17), the frequency spectra of the desired time-domain signals are obtained. Then, the desired timedomain signals $y_{r}$ are obtained by the inverse Fourier transformation on $Y$. These time-domain signals are referred to as reference signals because they are transformed from the reference spectra

$$
Y=L P \text {. }
$$

To obtain the drive loads for a test system, (13) should be generalized for both zero and non-zero initial condition cases. Thus, (13) is expressed in the form

$$
y_{k}=O z(k \cdot n)+H f_{k}
$$

where

$$
\begin{aligned}
& \mathrm{y}_{\mathrm{k}}=\left[\begin{array}{c}
\mathrm{y}_{\mathrm{r}}(\mathrm{k} \cdot \mathrm{n}) \\
\mathrm{y}_{\mathrm{r}}(\mathrm{k} \cdot \mathrm{n}+1) \\
\vdots \\
\mathrm{y}_{\mathrm{r}}(\mathrm{k} \cdot \mathrm{n}+(\mathrm{n}-1))
\end{array}\right] \text {, } \\
& \mathrm{O}=\left[\begin{array}{c}
\mathrm{C}_{\mathrm{d}} \\
\mathrm{C}_{\mathrm{d}} \mathrm{A}_{\mathrm{d}} \\
\vdots \\
\mathrm{C}_{\mathrm{d}} \mathrm{d}_{\mathrm{n}-1}
\end{array}\right] \\
& \mathrm{f}_{\mathrm{k}}=\left[\begin{array}{c}
\mathrm{f}(\mathrm{k} \cdot \mathrm{n}) \\
\mathrm{f}(\mathrm{k} \cdot \mathrm{n}+1) \\
\vdots \\
\mathrm{f}(\mathrm{k} \cdot \mathrm{n}+(\mathrm{n}-1))
\end{array}\right] \text {. }
\end{aligned}
$$

Any $n$-length driving forces can be represented as

$$
f_{k}= \begin{cases}\mathrm{H}^{-1} \mathrm{y}_{\mathrm{k}}, & \mathrm{k}=0, \\ \mathrm{H}^{-1}\left(\mathrm{y}_{\mathrm{k}}-\mathrm{Oz}(\mathrm{k} \cdot \mathrm{n})\right), & \mathrm{k}=1,2, \ldots,\end{cases}
$$

where $z$ can be obtained using (10) and the observability matrix $O$ is computed after the realization of the system. It is worth mentioning that the system transfer matrix $H$ should be handled by truncated singular value decomposition (TSVD). This truncation brings noise filtering for environmental testing. Details can be found in [19] and are not discussed in this article.

To obtain the inputs by (20) in a super-Gaussian test, we should transform the Gaussian reference signal $y_{r}$ into a super-Gaussian reference signal $\bar{y}_{r}$ with the specified kurtosis. The ZMNL functions we adopt in this article are

$$
\bar{y}_{r}=A \cdot \sinh \left(a \cdot y_{r}\right)
$$


where $A$ is used to rescale the PSD as changed by the ZMNL transformation. Note that the ZMNL transformation may cause magnitude distortion of the PSD if the dynamic range is large, according to Kihm et al. and Steinwolf $[6,9]$. To find suitable parameter a for the ZMNL transformation using (21), the MATLAB function fminsearch is recommended for a given kurtosis to locate the unknown parameters so that the error function

$$
E r=\left|K_{y, i}-K_{r, i}\right|
$$

is minimized. In (22), $K_{y, i}$ denotes the kurtosis of the $i^{\text {th }}$ block of the response signals and $K_{r, i}$ denotes the desired kurtosis of the $i^{\text {th }}$ block of the reference signals.

To summarize, the flow chart of the time-domain procedure for a super-Gaussian test is shown in Figure 3. First, reference spectra $R$ are decomposed by the Cholesky decomposition into $L$. The frequency spectra $Y$ of the reference signals for a Gaussian test are obtained using (17). Afterward, the Gaussian reference signal $\mathrm{y}_{\mathrm{r}}$ is gained by inverse Fourier transformation on $Y$. The super-Gaussian reference signal $\bar{y}_{\mathrm{r}}$ can be obtained using (21). To acquire the input signals $f$ for a super-Gaussian test, it is divided by the system transfer matrix $H$ as in (20). The input signals $f$ are then sent to stimulate the under-test articles, and the response signals $y$ of the under-test articles are gathered. In this step, the power spectra and kurtoses of $y$ are obtained and compared with their reference values. This loop continues until the power spectra $S_{y y}$ and the kurtoses $K_{y}$ converge to their references $R$ and $K_{r}$, respectively.

\section{WOA Method}

4.1. WOA Operation for the Gaussian Test. The time-domain randomization proposed by Smallwood and Paez [2] is divided into 2 parts. The first part is to convert one frame pseudorandom signal into a vast number of similar but different signals. The second part is to window and overlapadd these signals for the generation of infinite nonperiodic true random signals. To express our method for a superGaussian test, the original WOA operation for a Gaussian test is necessary to be introduced.

The windowing operation is used to mitigate leakage when we cut off a segment of signals for analysis. Frequently used window functions include the half-sine, Potter, and Hanning windows. In environmental testing, we typically use the half-sine window. Figure 4 demonstrates the schematic diagram of a windowing operation employing the half-sine window. After being windowed, the blocks of data are then overlapped and added to each other as outlined in Figure 5. In Figure 5, $n$ is the length of a frame of data. The $2^{\text {nd }}$ frame of data is delayed with respect to the $1^{\text {st }}$ frame by $n / 2$. Therefore, the overlap factor is 2 . The overlap factor $p$ is defined as the ratio of the block length $n$ to the number of

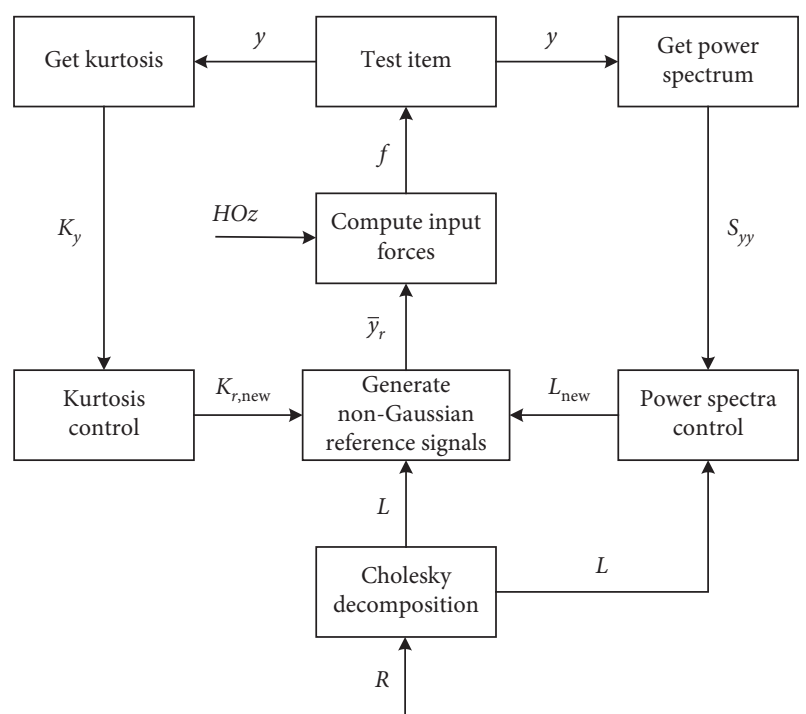

Figure 3: Flow chart of a super-Gaussian test procedure.

samples over which a block of data is delayed with respect to the previous block in the overlap-add operation. In this paper, we set this factor of all overlapping operations to be 2 .

4.2. WOA Operation for a Super-Gaussian Test. If the signals being processed by the WOA method follow a Gaussian distribution, the kurtoses of the signals are not changed. This conclusion has been verified by Steinwolf [4]. In fact, he also quantified how the kurtoses were changed by a half-sine window and overlap-add operation with the expression [4]

$$
K_{2}-3=\frac{3}{2 p}\left(K_{1}-3\right)
$$

where $K_{1}$ is the averaged kurtoses of all blocks of signals before the WOA operation, $K_{2}$ is the averaged kurtoses of all blocks of signals after the operation, and $p$ is the overlap factor. The military standard [20] stipulates we should gather at least 50 blocks of signals to acquire $K_{1}$ if we employ the arithmetical average. As mentioned previously, the overlap factor is 2 in this article, so (23) can be simplified to [4]

$$
K_{2}=\frac{3}{4} K_{1}+\frac{3}{4} \text {. }
$$

Steinwolf s equation (24) is deduced for super-Gaussian signals being processed but is also suitable for sub-Gaussian situations. However, it does not reveal how the kurtoses of the response signals are changed if we window and overlapadd the input voltage signals. Here, we explain the relationship based on (18).

The $k^{\text {th }}$ block of input signals $f_{k}$ and the $k^{\text {th }}$ block of super-Gaussian reference signals $\bar{y}_{\mathrm{k}}$ are related to the expression shown in (25) if the initial condition $z(k n)=0$. 


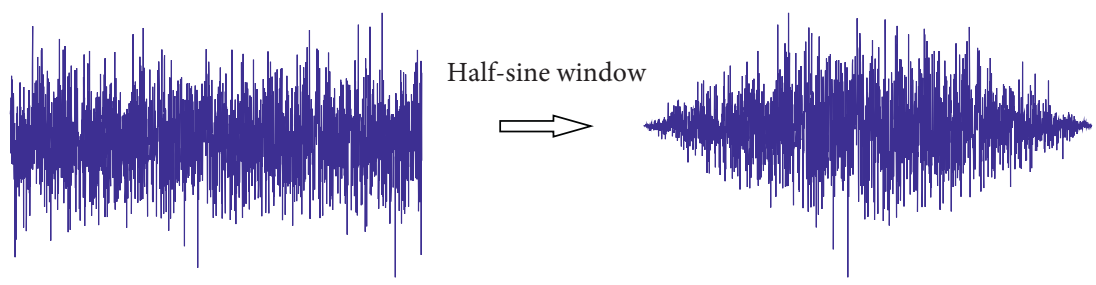

FIgURE 4: Schematic diagram of the windowing operation.

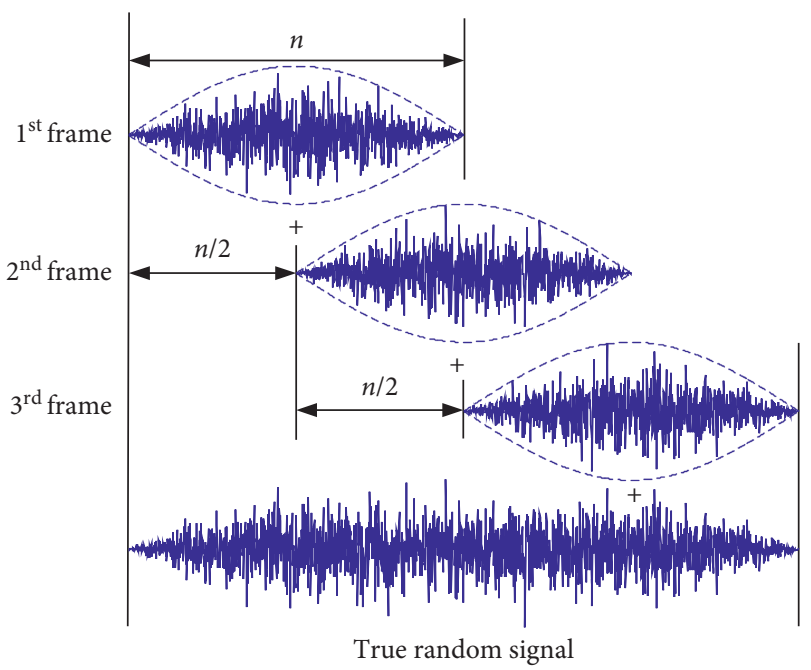

Figure 5: Schematic diagram of the overlap-add operation.

$$
\begin{aligned}
\overline{\mathrm{y}}_{\mathrm{k}}= & {\left[\begin{array}{c}
\overline{\mathrm{y}}_{\mathrm{r}}(\mathrm{k} \cdot \mathrm{n}) \\
\overline{\mathrm{y}}_{\mathrm{r}}(\mathrm{k} \cdot \mathrm{n}+1) \\
\vdots \\
\overline{\mathrm{y}}_{\mathrm{r}}(\mathrm{k} \cdot \mathrm{n}+(\mathrm{n}-1))
\end{array}\right]=\left[\begin{array}{cccc}
\mathrm{H}_{0} & 0 & \cdots & 0 \\
\mathrm{H}_{1} & \mathrm{H}_{0} & 0 & \vdots \\
\vdots & \vdots & \ddots & 0 \\
\mathrm{H}_{\mathrm{n}-1} & \mathrm{H}_{\mathrm{n}-2} & \cdots & \mathrm{H}_{0}
\end{array}\right] } \\
& \cdot\left[\begin{array}{c}
\mathrm{f}(\mathrm{k} \cdot \mathrm{n}) \\
\mathrm{f}(\mathrm{k} \cdot \mathrm{n}+1) \\
\vdots \\
\mathrm{f}(\mathrm{k} \cdot \mathrm{n}+(\mathrm{n}-1))
\end{array}\right] .
\end{aligned}
$$

If we window the input signals $f_{k}$, the windowed inputs are

$$
f_{k}^{\text {windowed }}=\left[\begin{array}{llll}
w_{1} & & & \\
& w_{2} & & \\
& & \ddots & \\
& & & w_{n}
\end{array}\right]\left[\begin{array}{c}
f(k \cdot n) \\
f(k \cdot n+1) \\
\vdots \\
f(k \cdot n+(n-1))
\end{array}\right],
$$

where $w_{n}$ represents the window function. Substituting (26) into (25), we have

$$
\begin{aligned}
& H f_{k}^{\text {windowed }}=\left[\begin{array}{cccc}
H_{0} & 0 & \cdots & 0 \\
H_{1} & H_{0} & 0 & \vdots \\
\vdots & \vdots & \ddots & 0 \\
H_{n-1} & H_{n-2} & \cdots & H_{0}
\end{array}\right]\left[\begin{array}{llll}
w_{1} & & & \\
& w_{2} & \\
& & \ddots & \\
& & & w_{n}
\end{array}\right] \\
& {\left[\begin{array}{c}
f(k \cdot n) \\
f(k \cdot n+1) \\
\vdots \\
f(k \cdot n+(n-1))
\end{array}\right]} \\
& =\left[\begin{array}{cccc}
w_{1} & & & \\
& w_{2} & & \\
& & \ddots & \\
& & & w_{n}
\end{array}\right]\left[\begin{array}{cccc}
H_{0} & 0 & \cdots & 0 \\
H_{1} & H_{0} & 0 & \vdots \\
\vdots & \vdots & \ddots & 0 \\
H_{n-1} & H_{n-2} & \cdots & H_{0}
\end{array}\right] \\
& {\left[\begin{array}{c}
f(k \cdot n) \\
f(k \cdot n+1) \\
\vdots \\
f(k \cdot n+(n-1))
\end{array}\right]} \\
& =\left[\begin{array}{cccc}
w_{1} & & & \\
& w_{2} & & \\
& & \ddots & \\
& & & w_{n}
\end{array}\right]\left[\begin{array}{c}
\bar{y}_{r}(k \cdot n) \\
\bar{y}_{r}(k \cdot n+1) \\
\vdots \\
\bar{y}_{r}(k \cdot n+(n-1))
\end{array}\right] \\
& =\bar{y}_{k}^{\text {windowed }} \text {. }
\end{aligned}
$$

From (27), we know that the window operation on the input signals is equivalent to the same window operation on the response signals.

Then, we study the overlap-add operation between the $k^{\text {th }}$ block of the signals and the $(k+1)^{\text {th }}$ block of the signals. First, we delay the $(k+1)^{\text {th }}$ block of the input signal $f_{k+1}$ by $n /$ 2 and add the corresponding segment of $f_{k+1}^{\text {delayed }}$ to $f_{k}$. The overlap-added input signal is 


$$
f_{k}^{\text {overlap-added }}=f_{k}+f_{k+1}^{\text {delayed }}=\left[\begin{array}{c}
f(k \cdot n) \\
f(k \cdot n+1) \\
\vdots \\
\left.f(k \cdot n)+\left(\frac{n}{2}-1\right)\right) \\
f\left(k \cdot n+\frac{n}{2}\right) \\
\vdots \\
f((k \cdot n)+(n-1))
\end{array}\right]+\left[\begin{array}{c}
0 \\
0 \\
\vdots \\
0 \\
f((k+1) \cdot n) \\
\vdots \\
f\left((k+1) \cdot n+\left(\frac{n}{2}-1\right)\right)
\end{array}\right]
$$

Substituting (28) into (25), the equation is

$$
H\left(f_{k}+f_{k+1}^{\text {delayed }}\right)=\bar{y}_{k}+H f_{k+1}^{\text {delayed }} .
$$

Because the first $n / 2$ rows of $\mathrm{f}_{\mathrm{k}+1}^{\text {delayed }}$ are zeros, the term $\mathrm{Hf}_{\mathrm{k}+1}^{\text {delayed }}$ can be simplified as

$$
\begin{aligned}
& H f_{k+1}^{\text {delayed }}=\left[\begin{array}{cccccc}
0 & \cdots & 0 & 0 & \cdots & 0 \\
\vdots & \ddots & \vdots & \vdots & & \vdots \\
0 & \cdots & 0 & 0 & \cdots & 0 \\
0 & \cdots & 0 & H_{0} & \cdots & 0 \\
\vdots & & \vdots & \vdots & \ddots & \vdots \\
0 & \cdots & 0 & H_{((n / 2)-1)} & \cdots & H_{0}
\end{array}\right]\left[\begin{array}{c}
0 \\
\vdots \\
0 \\
f((k+1) \cdot n) \\
\vdots \\
f\left((k+1) \cdot n+\left(\frac{n}{2}-1\right)\right)
\end{array}\right] \\
& =\left[\begin{array}{c}
0 \\
\vdots \\
0 \\
{\left[\begin{array}{ccc}
H_{0} & 0 & 0 \\
\vdots & \ddots & 0 \\
H_{((n / 2)-1)} & \cdots & H_{0}
\end{array}\right]\left[\begin{array}{c}
f((k+1) \cdot n) \\
\vdots \\
f\left((k+1) \cdot n+\left(\frac{n}{2}-1\right)\right)
\end{array}\right]}
\end{array}\right] .
\end{aligned}
$$


The $(k+1)^{\text {th }}$ block of the input signals $f_{k+1}$ and the $(k+1)$ th block of the super-Gaussian reference signals $\bar{y}_{k+1}$ are related to the expression in (29) if the initial condition $z((k+1) n)=0$ :

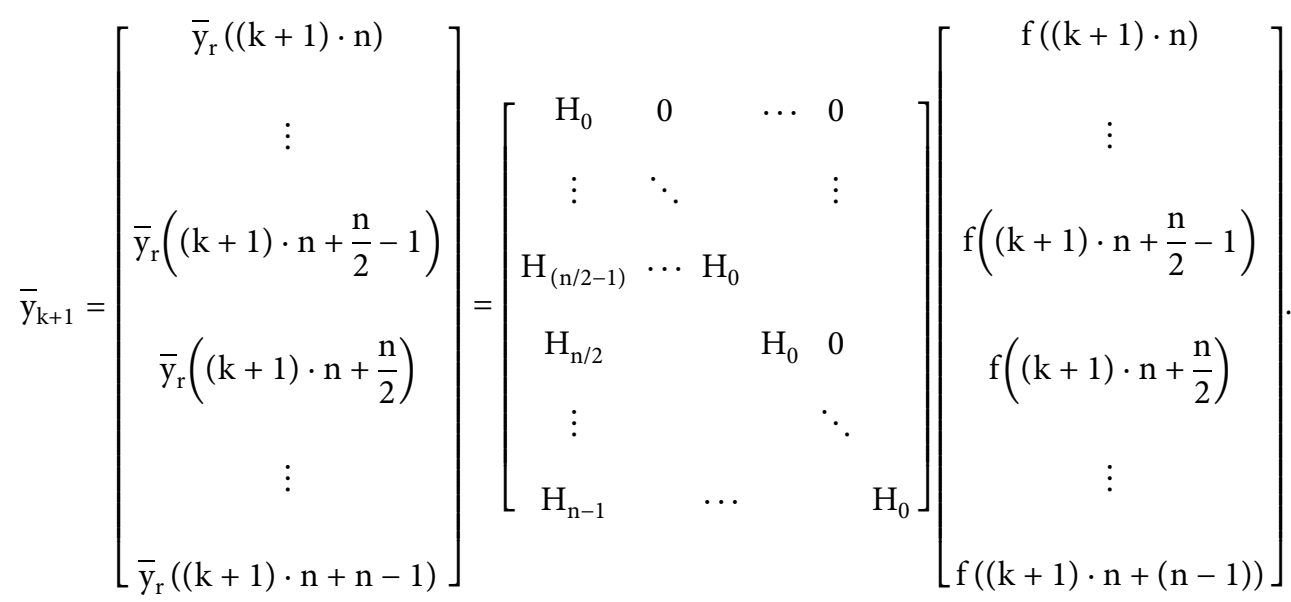

As seen from (31), the non-zero rows of the right term in (30) correspond to the first $n / 2$ rows of the right term of (31). As a result, (30) can be expressed as

$$
H f_{k+1}^{\text {delayed }}=\left[\begin{array}{c}
0 \\
\vdots \\
0 \\
\bar{y}_{r}((k+1) \cdot n) \\
\vdots \\
\bar{y}_{r}\left((k+1) \cdot n+\left(\frac{\mathrm{n}}{2}-1\right)\right)
\end{array}\right]=\bar{y}_{k+1}^{\text {delayed }},
$$

and with (32), (29) becomes

$$
\bar{y}_{k}+\bar{y}_{k+1}^{\text {delayed }}=H\left(f_{k}+f_{k+1}^{\text {delayed }}\right) \text {. }
$$

Equation (33) indicates that the overlap-add operation on input signals is equivalent to the same operation on the response signals.

To summarize, as shown in (30) and (33), we find that if we apply the WOA operation on the voltage signals of a test system, the responses of the shaker table are transformed in the same way. This conclusion is reached under the assumption that the initial condition of every block signal is zero. Numerical simulation shows that omitting the initial conditions in (20) does not change the response spectra or response kurtoses. Therefore, when we study the response kurtoses, the effects of the initial conditions can be neglected. Since the system Markov parameters and the frequency response functions all represent the same dynamic system, the above conclusion is expected to apply to environmental testing using both the time-domain and frequency-domain procedures. This conclusion will be verified in the numerical simulation.

Leaving out other factors that may change the response kurtoses, under ideal conditions, the response kurtoses should be equal to the reference kurtoses if WOA operation is not applied. Once the WOA is applied on the input voltage signals in a super-Gaussian test, according to (30) and (33), the response kurtoses can be anticipated based on Steinwolf's equation [4]. When the window function is a half-sine window and the overlap factor is 2 , the kurtoses of the response signals are

$$
K_{y}=\frac{3}{4} K_{r}+\frac{3}{4},
$$

where $K_{y}$ denotes the averaged kurtoses of the response signals and $K_{r}$ represents the desired reference kurtoses.

4.3. Numerical Example. In this finite element simulation, the WOA operation is introduced into both the time-domain and frequency-domain super-Gaussian test procedures. The timedomain procedure is outlined in Figure 3. The frequencydomain procedure utilizes a frequency response function to compute the input voltage signals, which can be found in [3]. As seen in Figure 6, a model of a cantilever beam consists of 5 plane beam elements whose nodes have one translational degree of freedom and one rotational degree of freedom. The reference spectrum of the simulation is described in Table 1. The parameters of the Bernoulli-Euler beam are listed in Table 2. Above all, the damping of this structure is defined in terms of (35), where $\zeta_{k}$ is the $k^{\text {th }}$ modal damping ratio; $M_{k}$ and $K_{k}$ are the $k^{\text {th }}$ modal mass and stiffness, respectively. To eliminate the impact of damping on the response kurtoses, the modal damping ratio of every mode is set to 0.05 in this simulation. The reference spectra are still those described in Table 1.

$$
\mathrm{C}_{\mathrm{r}}=2 \zeta_{\mathrm{r}} \sqrt{\mathrm{M}_{\mathrm{r}} \mathrm{K}_{\mathrm{r}}}
$$




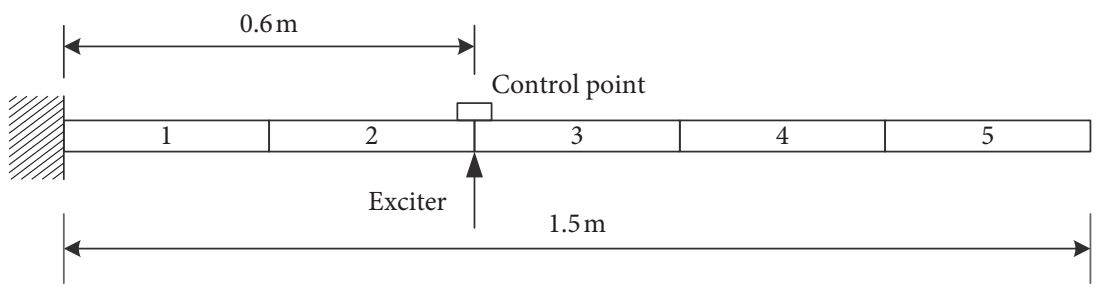

Figure 6: Diagram of the simulated cantilever beam.

TABle 1: Parameter setting for reference spectrum.

\begin{tabular}{lc}
\hline Frequency $(\mathrm{Hz})$ & Reference spectrum $\left(10^{-6} \mathrm{~g}^{2} / \mathrm{Hz}\right)$ \\
\hline 20 & 0.2 \\
100 & 1 \\
1000 & 1 \\
2000 & 0.5 \\
\hline
\end{tabular}

TABLe 2: Parameters of the cantilever beam model.

\begin{tabular}{lc}
\hline Physical and geometrical properties & Values \\
\hline Young's modulus $(\mathrm{GPa})$ & 206 \\
Density $\left(\mathrm{kg} / \mathrm{m}^{3}\right)$ & 7820 \\
Length $(\mathrm{m})$ & 1.5 \\
Width $(\mathrm{m})$ & 0.06 \\
Height $(\mathrm{m})$ & 0.02 \\
Sampling frequency $(\mathrm{Hz})$ & 5120 \\
\hline
\end{tabular}
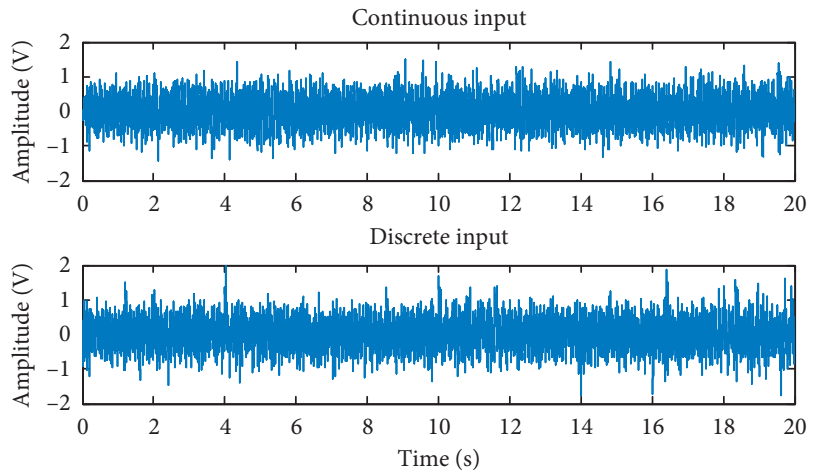

FIgURE 7: Continuous input voltage and discrete input voltage.

First, we compute the discrete input using (20) and apply the WOA operation to the input. The continuous input and discrete input are both shown in Figure 7. From the figure, we can see that there are more high-excursion peaks in the discrete input voltages than in the continuous input voltages. The sampling period is $0.4 \mathrm{~s}$, and the positions of these peaks are located at the frame boundaries of the discrete input. It is obvious that the WOA operation can remove discontinuity at the adjacent area of different frames. Figure 8 shows the autopower spectral density (ASD) of the control point generated by the continuous input, and Figure 9 demonstrates the corresponding kurtoses of all 50 frames of responses. The reference kurtosis of the simulation is 10 , and the averaged kurtosis of the response is 8.05, which fits (34). To further validate the relationship between the reference

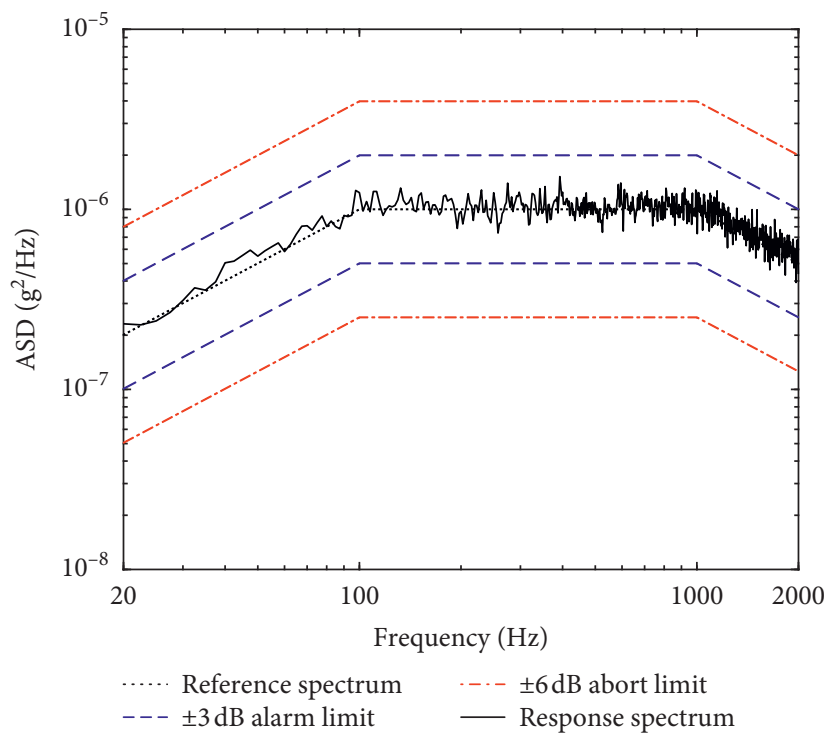

FIGURE 8: ASD of the control point.

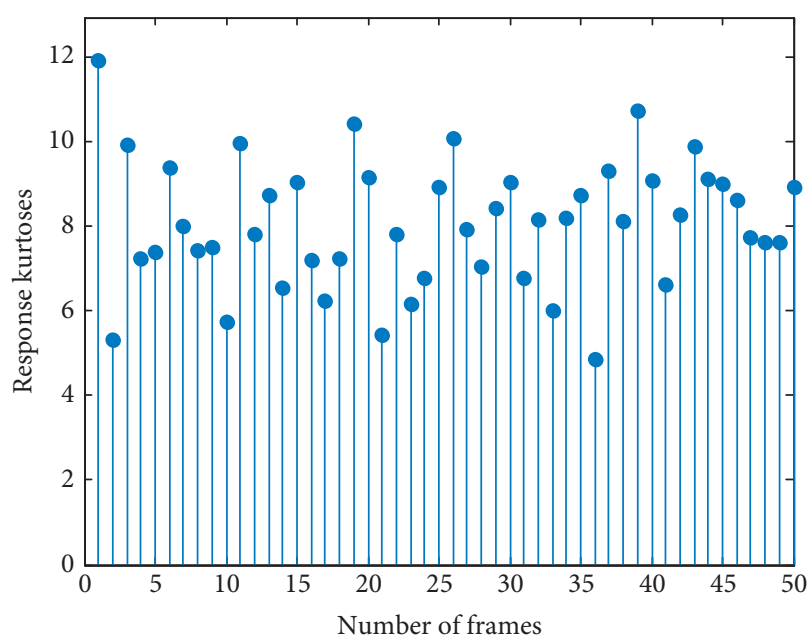

FIgURE 9: Kurtoses of all 50 frames of the response.

kurtosis and the averaged response kurtosis, the averaged response kurtoses are shown in Figure 10 with the change of the reference kurtoses. The solid curve in this figure represents (34), the plus symbols denote the results from the time-domain procedure, and the stars show the results from the frequency-domain procedure. The three lines match well when the reference kurtoses are below 20, which confirms 


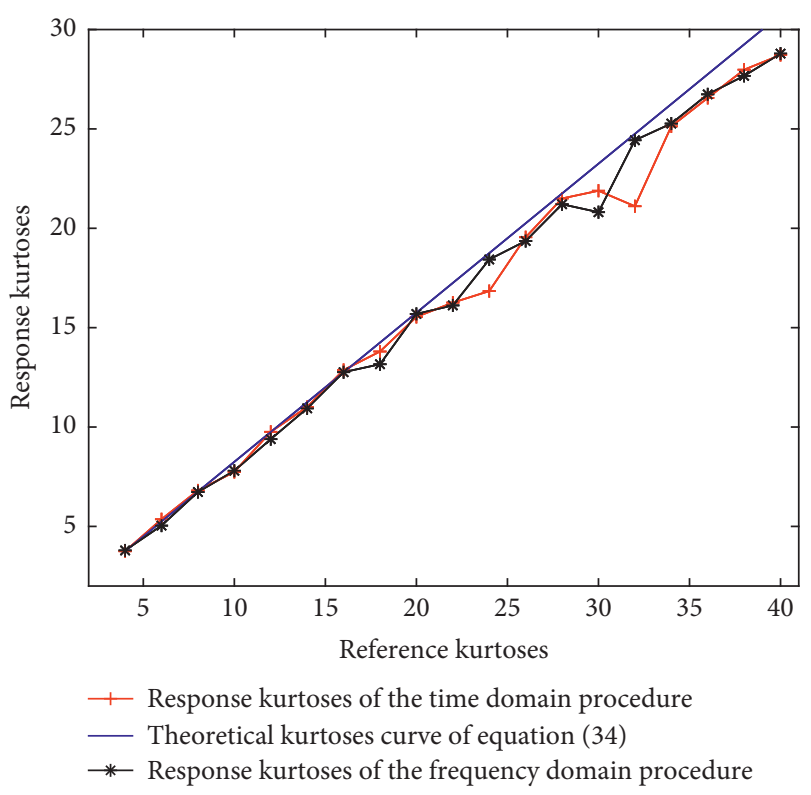

Figure 10: Numerical relation between the reference kurtoses and the response kurtoses: theoretical curve based on equation (34) (solid line); averaged kurtoses of the response using the timedomain procedure (broken line with plus signs); averaged kurtoses of the response using frequency-domain procedure (broken line with stars).

that (34) is credible to some degree. When the reference kurtoses are above 20, the averaged response kurtoses from both procedures deviate from the theoretical curve. Nevertheless, the overall trend of the averaged response kurtoses fits that of (34), and most of the deviations are acceptable. Although the influences of the WOA operation on response kurtoses are deduced using the state-space model, the conclusion is also valid using the frequency-domain approach of computing the inputs.

\section{The Improved Method for a Super-Gaussian Test with a Continuous Input}

Knowing the relationship between averaged response kurtosis and the reference kurtosis with the WOA operation applied, we seem to be able to simply employ (34) to reset the reference kurtosis $K_{r}^{\prime}$ so that the averaged response kurtosis $K_{y}$ can be equal to the true reference kurtosis $K_{r}$ with the expression

$$
K_{r}^{\prime}=\frac{4}{3} K_{r}+1
$$

where $K_{r}^{\prime}$ is used in (22) to generate the reference signals. However, (34) is not valid to all kinds of super-Gaussian signals. The kurtosis of the signal operated by WOA method is highly related with the position of the high-excursion peaks in the signal. The support of the conclusion will be introduced in this section. Together, an improved method is recommended in this section to eliminate the kurtosis reduction caused by the WOA operation. The method starts by studying the reason for the kurtosis reduction.
5.1. Kurtosis Reduction Induced by the WOA Operation. According to the conclusion we draw in Section 4.2, the WOA operation on the input is equivalent to the same operation on the response. Under ideal conditions, the response should be equal to the reference signals we build at the beginning. To determine the reason for the kurtosis reduction induced by the WOA operation, we can apply the WOA operation on the reference signals. Figure $11 \mathrm{dem}-$ onstrates the process of the windowing operation on one frame of the reference signals using a half-sine function. Intuitively, super-Gaussian signals are characterized by high-excursion peaks. The number and heights of these peaks are highly related to the kurtoses of the signals. In Figures 11 and 12, the $X$ coordinates denote time, and the $Y$ coordinates represent the amplitude of the signal. The sampling period of one frame of the signal is $0.4 \mathrm{~s}$, which indicates that the point in Figure 11 with $X=0.2117$ is located in the middle area of the signal. After windowing, the amplitudes of the high peaks in the middle area of the signal decrease much less than those at either ends. Figure 12 outlines the OLA operation between the first windowed frame and the second windowed frame. Only the $n$-length signal after an OLA operation is provided in the figure. Because the second frame of the windowed signal is delayed by $n / 2$ with respect to the first frame of the windowed signal, the middle area of the first frame of the windowed signal corresponds to the left end of the second frame. Both ends of the windowed signals have small amplitudes, which causes the high peaks in the middle area of the first frame to barely change by the adding operation. In contrast, the peaks in the right end of the first frame correspond to the peaks with large amplitudes of the second frame, and the same applies in the middle area, resulting in large changes to the peak amplitudes at the end. As a result, if the high-excursion peaks are distributed in the marginal area of every frame of the signals, the highexcursion peaks are reduced in number by the WOA operation, and kurtoses of the windowed and overlapadded signals may be substantially decreased. There were several methods that generate the super-Gaussian signal with specified locations of high-excursion peaks. For example, an on-band phase manipulation method [21] creates the super-Gaussian signal with only one high peak per data block at specified locations. A method recommended by Zhang et al. [22] also have the same feature. To prevent the high-excursion peaks and kurtoses from being decreased by the WOA operation, we should move the high-excursion peaks' location.

5.2. Windowed ZMNL Transformation. The ZMNL transformation employs specific functions to transform a Gaussian signal into a super-Gaussian signal. The theory of this transformation is to maintain most of the data of the Gaussian signal and to amplify the data with relatively large amplitudes. The ZMNL function has the following property:

$$
\lim _{x \rightarrow 0} \frac{f(x)}{x}=1
$$




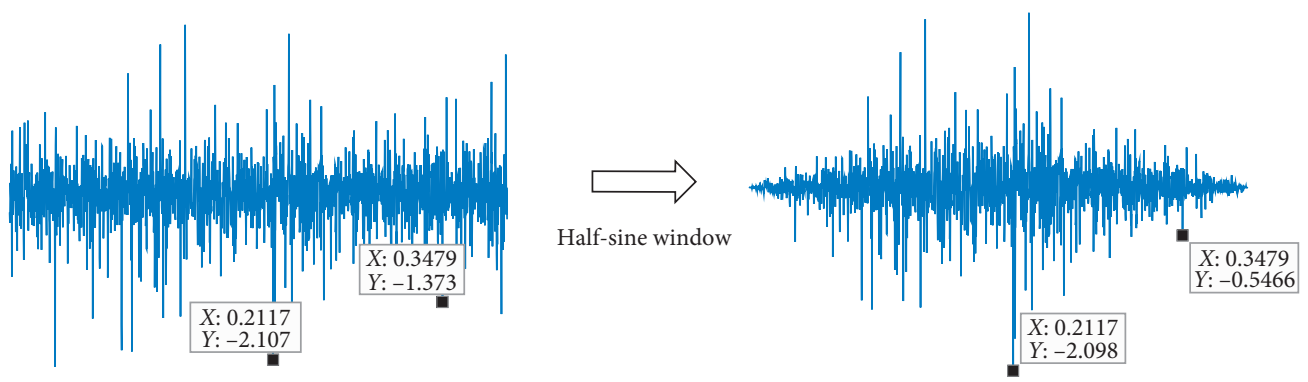

FIgURE 11: Windowing operation on the reference signals.

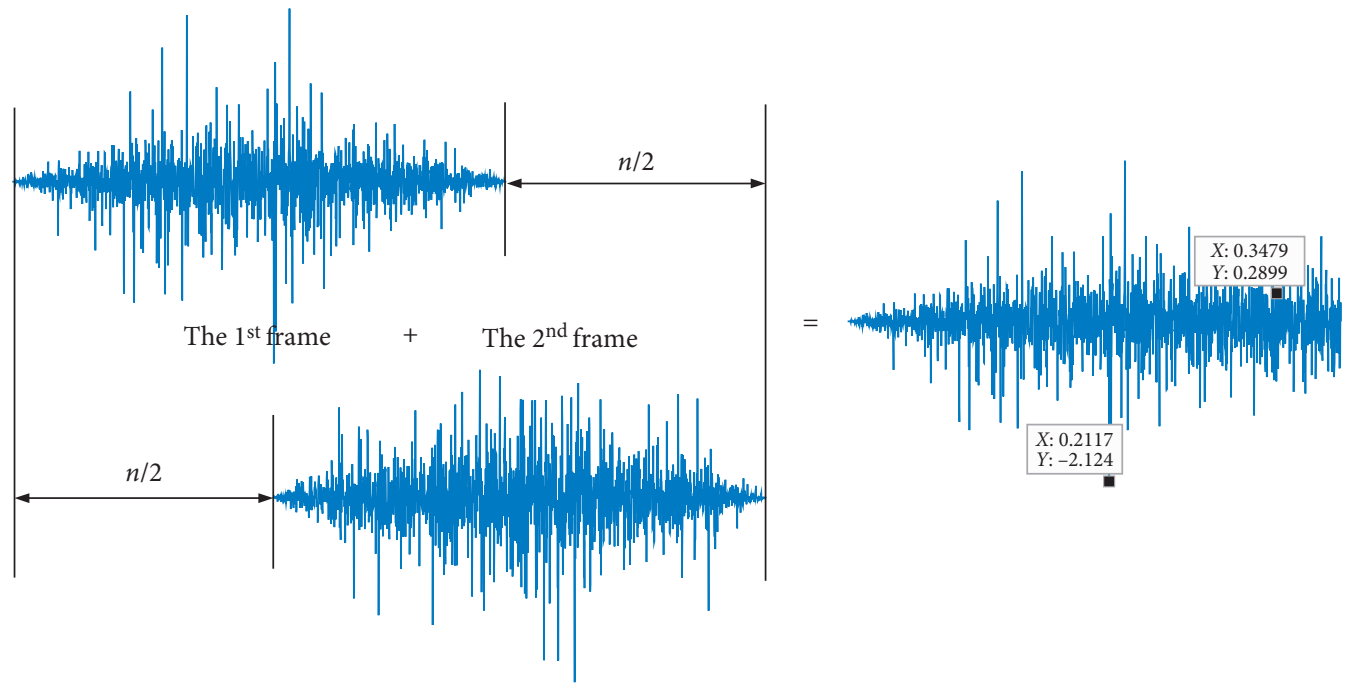

FIGURE 12: OLA operation on the reference signals.

where $f(x)$ denotes the ZMNL function and $x$ represents the data of a Gaussian signal. When $x$ approaches zero, the data are incorporated into the new super-Gaussian signal. Otherwise, $x$ values with large amplitudes are increased, and high-excursion peaks appear in a super-Gaussian transformation. Based on this property, we can shrink the amplitude of a Gaussian signal in the area near both ends with the window function

$$
w(t)= \begin{cases}1, & t \in\left(\frac{n}{2}-c, \frac{n}{2}+c\right), \\ b, & t \in\left[\frac{n}{2}+c, n\right] \text { or }\left[1, \frac{n}{2}-c\right],\end{cases}
$$

where we use $c$ to control the area that the high peaks generate; $b$ is less than 1 and is used to decrease the amplitudes of the data outside the central area. The original Gaussian signal is multiplied by (38) to reshape the amplitude of the signal. Then, we process this windowed signal with the ZMNL transformation. The ZMNL function we use in this article is the hyperbolic sine function. The expression of the transformation is

$$
\bar{y}_{r}=\frac{A \cdot \sinh \left(a \cdot w(t) \cdot y_{r}\right)}{w(t)} .
$$

To make the kurtosis of $\bar{y}_{\mathrm{r}}$ equal to the reference kurtosis, we need to find a suitable $a$ and $A$ so that $E r$ in (22) is minimized. Equation (38) is able to decrease the amplitude of $y_{r}$ in the marginal area of one frame signal. Because the amplitudes of the windowed signal in the middle positions are relatively large, it is more likely that the high-excursion peaks are created in the central positions. A schematic diagram of this process is given in Figure 13.

Because the mechanism of creating non-Gaussian features is similar for all types of ZMNL transformation, the method proposed in this section is suitable for any ZMNL function. It is worth mentioning that the method recommended by Zhang et al. [22] is also capable of adjusting the positions and the numbers of high-excursion peaks.

5.3. Kurtosis Increase Induced by the WOA Operation. Steinwolf's equation is deduced under the assumption that the high-excursion peaks are produced with random positions. If we can move these peaks to the middle area of every block of signals, as shown in Figure 13, the OLA method might increase the kurtoses. If the overlap factor is 2 , the OLA method delays the next frame of the signal by $n / 2$ and adds these signals together in a frame-by-frame form. Since the high peaks are moved to the middle, the interval between two adjacent high peaks is decreased to approximately $n / 2$, 


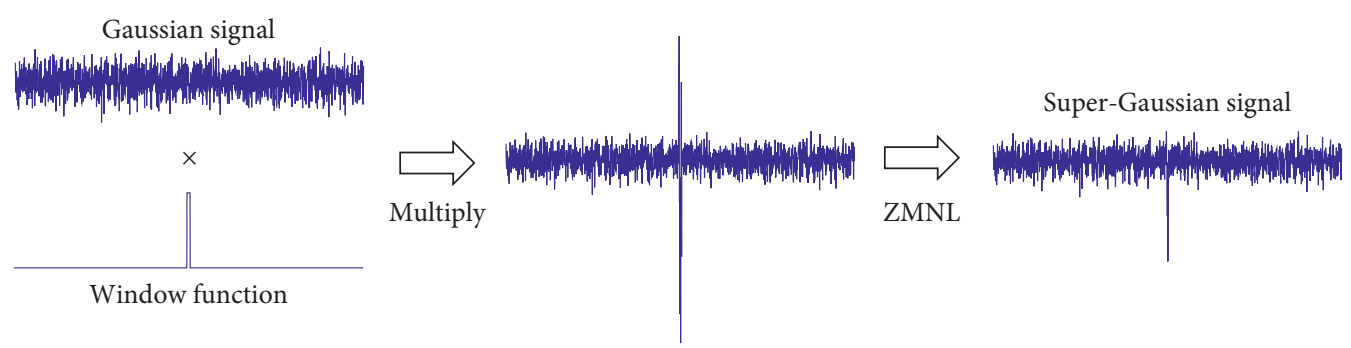

FIGURE 13: Schematic diagram of the improved ZMNL transformation.

less than the signal before the WOA operation. In addition, because the peaks are in the middle, the windowing operation barely changes the amplitude of these peaks. In general, the density of these high peaks is increased, and their amplitudes are nearly unchanged. The kurtoses of every block of the processed signals are increased.

To explain the issue intuitively, a numerical example is given. A signal is processed with the windowed ZMNL method first, and the processed signal is then windowed with the half-sine function and overlap-added. The signals before and after the WOA operation are presented in Figure 14. From the upper figure, we can see that the time interval of high peaks is $0.4 \mathrm{~s}$, which is also the sampling time of one frame signal. The time interval indicates that the windowed ZMNL transformation can move high-excursion peaks to the middle of every frame of signals. The figure below shows the signal after the WOA operation. The time interval is approximately $0.2 \mathrm{~s}$, which is half of the sampling time of one frame signal. This result suggests that the WOA method can increase the density of high-excursion peaks. The kurtosis of the signal before the WOA operation is 8 , as shown in Figure 15. From the figure below, we can see that the kurtoses of the signal after the WOA operation are increased substantially.

5.4. Discussion. According to the conclusions reached in the previous sections, we can see that the kurtosis of the signal after the WOA operation changes with respect to the positions of the high peaks. If these peaks are distributed randomly, the resulting kurtosis can be obtained using (34). The kurtosis gained by (34) is not the minimum kurtosis that the WOA method can create. By moving all the high peaks to both ends of every frame of signals, the minimum kurtosis can be reduced to 3 . Since the window function proposed in (38) amplifies the signal in the middle instead of at both ends, the windowed ZMNL transformation in Section 5.2 is not capable of reducing the kurtosis to 3 . By adjusting the parameters $b$ and $c$ of (38), the distribution of high peaks can be adjusted, and any value of output kurtosis in its interval can be achieved. The minimum value of the interval can be obtained using (34). When $b$ and $c$ are 0.1 and 3 in (38), the maximum averaged kurtoses obtained from a simulation are listed in Table 3.

In the experiment, we aim to make the response kurtoses equal to their preset references, which can also be achieved by adjusting $b$ and $c$. A simulation is given for this purpose.
In this simulation, we still use the same parameter settings described in Section 4.3. $c$ and $b$ in (38) are set to 50 and 0.65, respectively. First, we set the reference kurtosis to be 10 . The proposed ZMNL transformation is used to produce superGaussian signals with high peaks located in the middle, and the WOA operation is applied on the inputs in a frame-byframe form. The super-Gaussian response, its ASD, and the kurtoses of all 50 blocks of signals are demonstrated in Figures 16-18, respectively. The auto PSD of the response provided in Figure 18 indicates that the improved ZMNL transformation does not disturb the power spectrum. The mean value of the kurtoses shown in Figure 17 is 9.93, almost equal to its reference kurtosis. For further validation, a certain range of reference kurtoses and the corresponding averaged response kurtoses are documented in Figure 19, as in Figure 11. The averaged response kurtoses of the simulation are nearly identical to the reference kurtoses, and the figure indicates that the improved ZMNL transformation can eliminate the kurtosis reduction brought by the WOA operation regardless of the type of procedure employed.

The kurtosis increase caused by the WOA method can also be used to compensate for the kurtosis loss induced by other factors, for example, low damping. To examine the low damping effect, the damping coefficient is set to $0.2 \%$. When the windowed ZMNL transformation is not applied and the WOA method is applied to the input signal, the response kurtoses are as outlined in Figure 20. The reference kurtosis is 12, and the averaged kurtosis in Figure 20 is only 6.4, less than 9.75 (the kurtosis value obtained by (34)). When the windowed ZMNL transformation is applied with $b=0.4$ and $c=20$, the response kurtoses are as shown in Figure 21. The averaged kurtosis in Figure 21 is 11.5, nearly identical to the reference kurtosis.

\section{Experimental Validation}

The windowed ZMNL transformation has been validated in the numerical simulation; in addition, an experiment is conducted on a vibration table with two inputs and two outputs. In the experiment, two sensors are mounted on a vibration table. Each of them is used to collect the acceleration of different directions. We employ NI USB 6363 to send input voltage signals to the vibration table, and the voltage signals are acquired at the same time also by NI USB 6363. The data acquisition software is coded with LabVIEW and implemented on a computer. The reference kurtoses of the two points are where sensors are located at 5 and 9, 


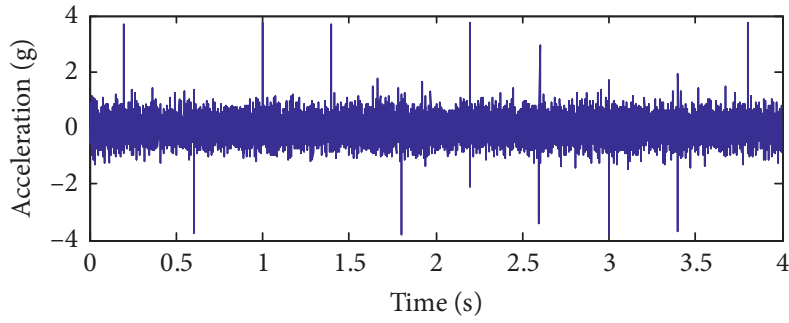

(a)

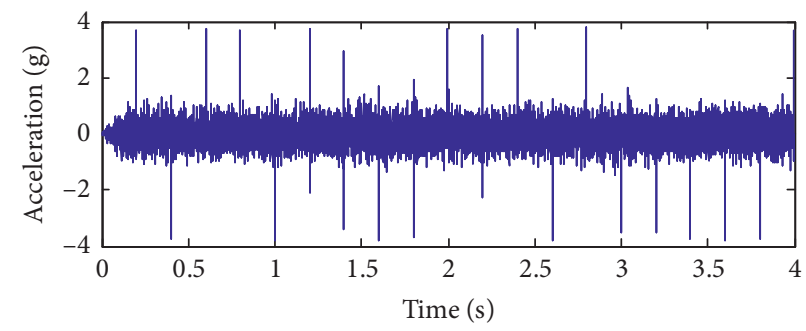

(b)

Figure 14: The signal before the WOA operation (a) and the signal after the WOA operation (b).

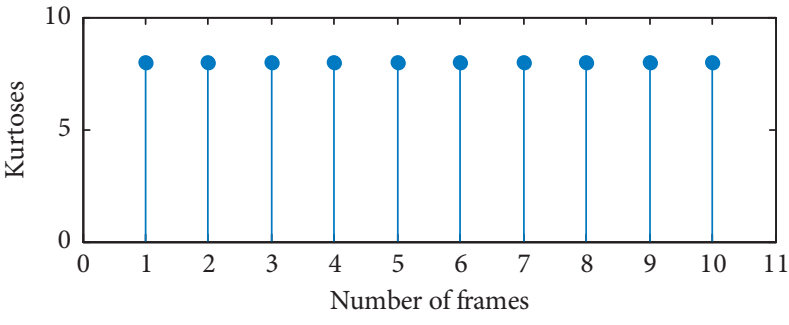

(a)

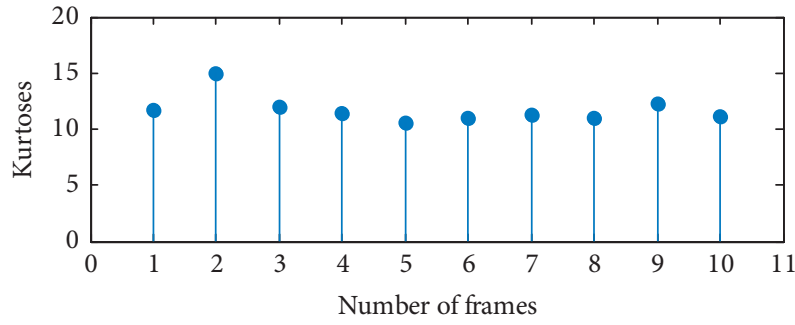

(b)

FIgURE 15: The kurtoses of the signal before the WOA operation (a) and the kurtoses of the signal after the WOA operation (b).

TABLE 3: The maximum kurtoses with the signal processed by the windowed ZMNL transformation.

\begin{tabular}{lc}
\hline Kurtoses of the signal before the WOA operation & Kurtoses of the signal after the WOA operation \\
\hline 4 & 4.48 \\
6 & 7.78 \\
8 & 11.33 \\
10 & 14.09 \\
12 & 17.29 \\
16 & 19.93 \\
\hline
\end{tabular}

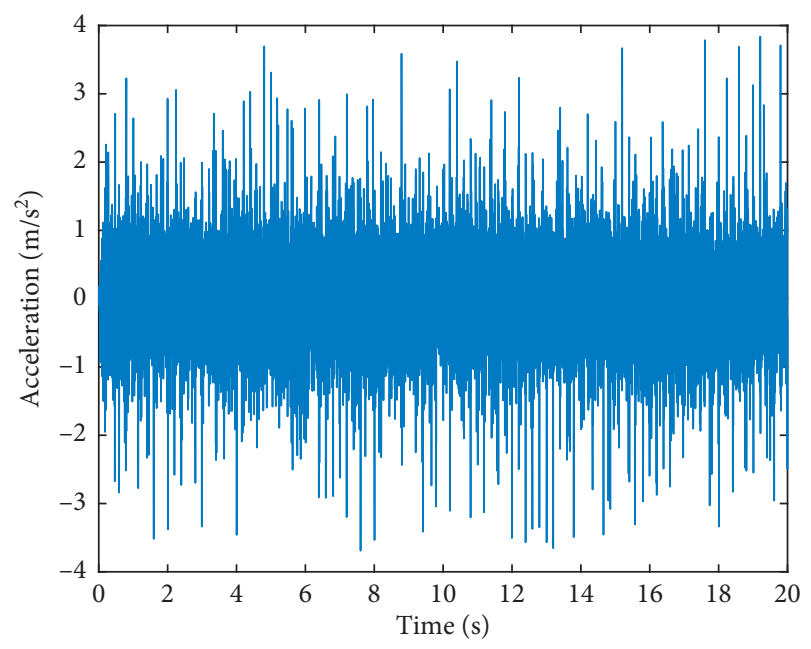

FIGURE 16: Fifty blocks of super-Gaussian responses with high peaks relocated in the middle of every frame.

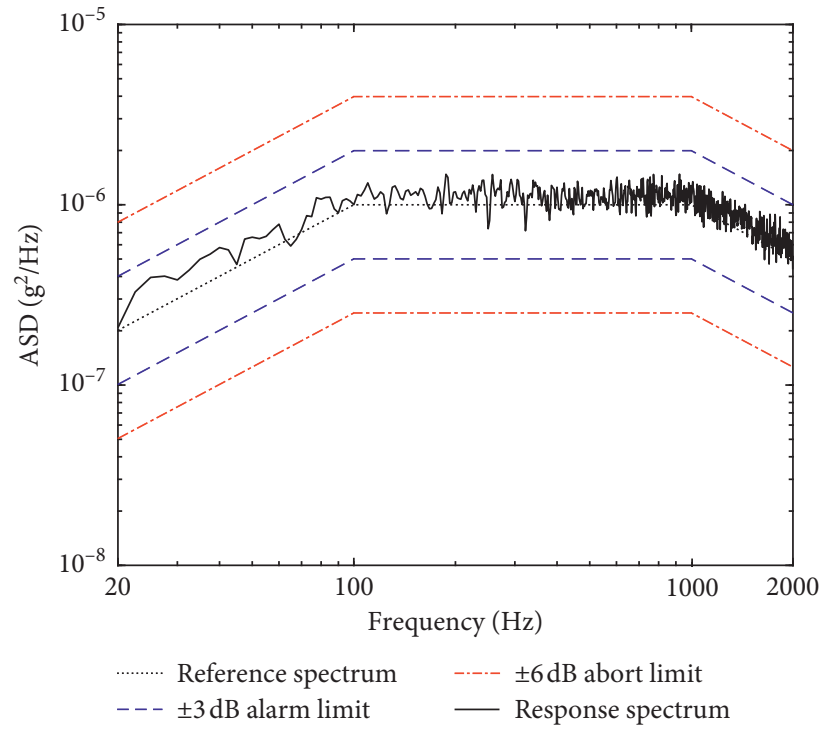

FIGURE 17: ASD of the super-Gaussian response. 


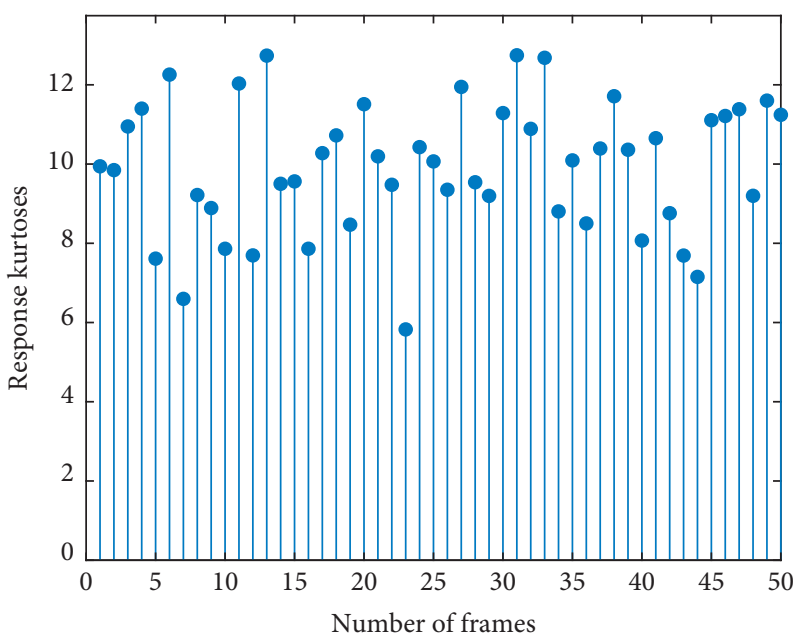

Figure 18: Kurtoses of all 50 blocks of the super-Gaussian response.

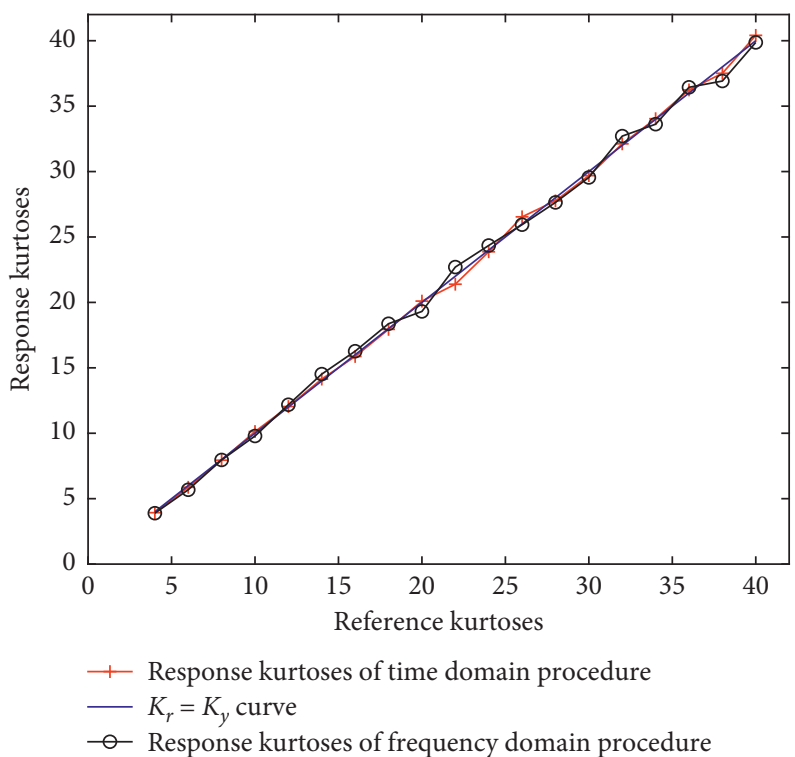

FIGURE 19: Numerical relationship between the reference kurtoses and the response kurtoses with the application of the proposed window function: $K_{r}=K_{y}$ curve (solid line); averaged kurtoses of the response generated by the time-domain procedure (broken line with plus signs); averaged kurtoses of the response generated by the frequency-domain procedure (broken line with circles).

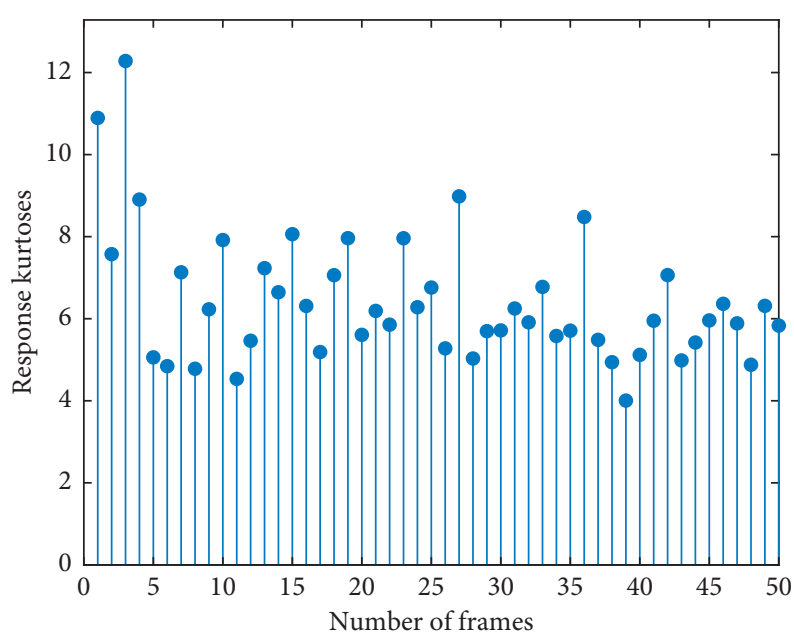

FIgURE 20: Response kurtoses when the WOA method is applied to the input signals. 


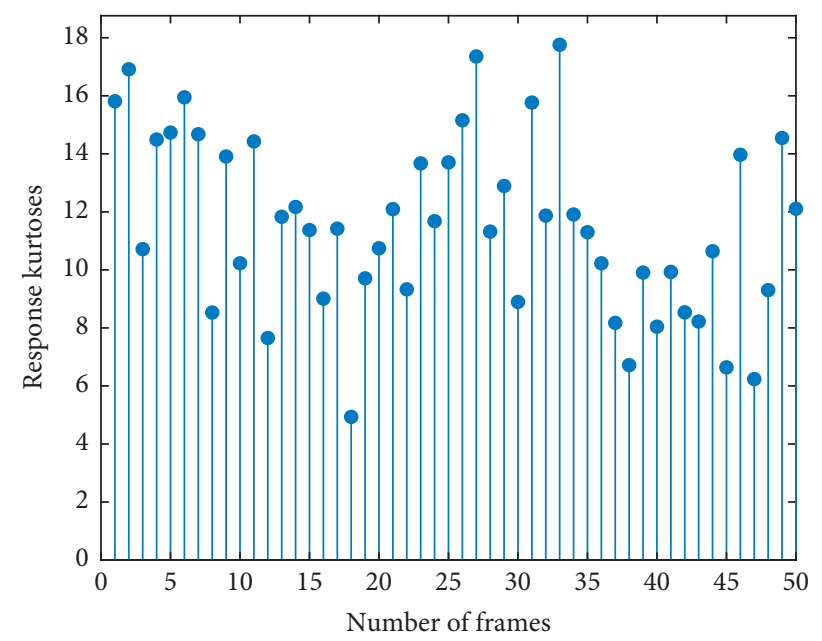

FIGURE 21: Response kurtoses when the windowed ZMNL transformation and WOA method are both applied.

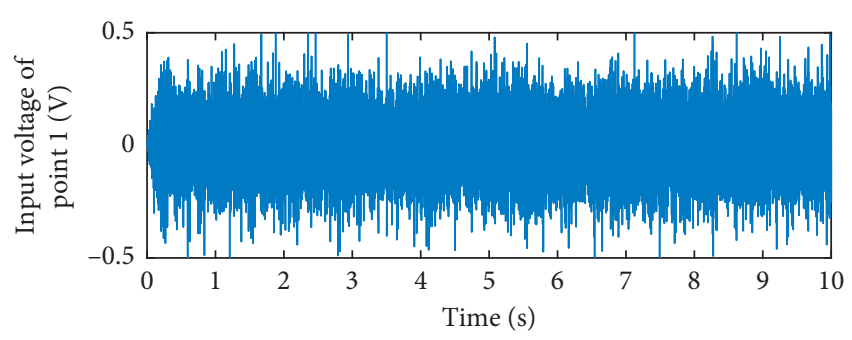

(a)

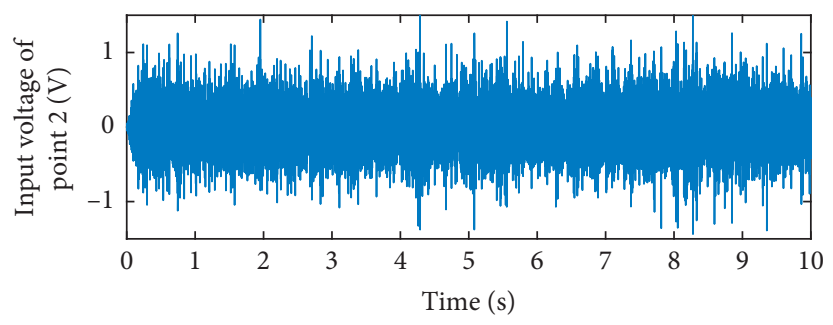

(b)

FiguRE 22: Input voltage signals sent to the vibration table.

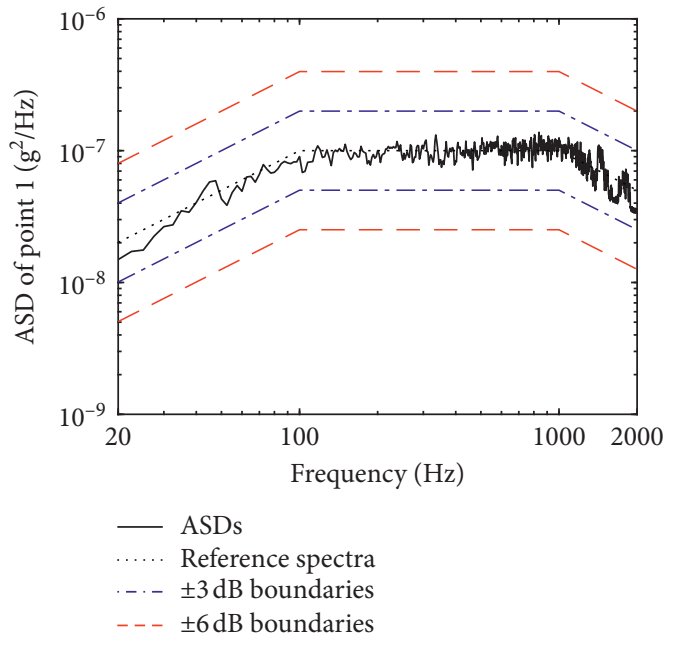

(a)

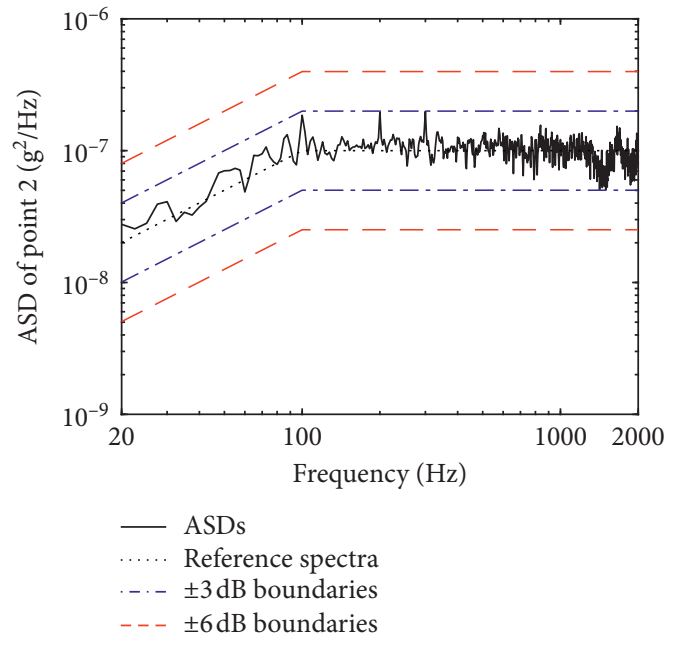

(b)

FIGURE 23: Continued. 


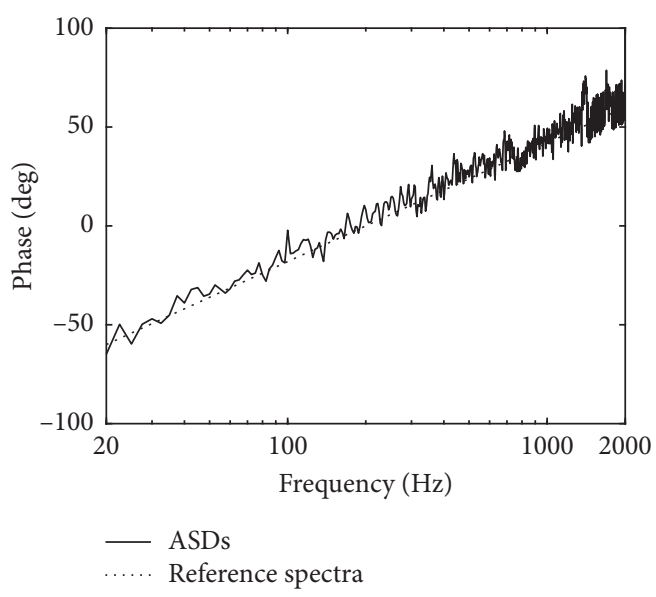

(c)

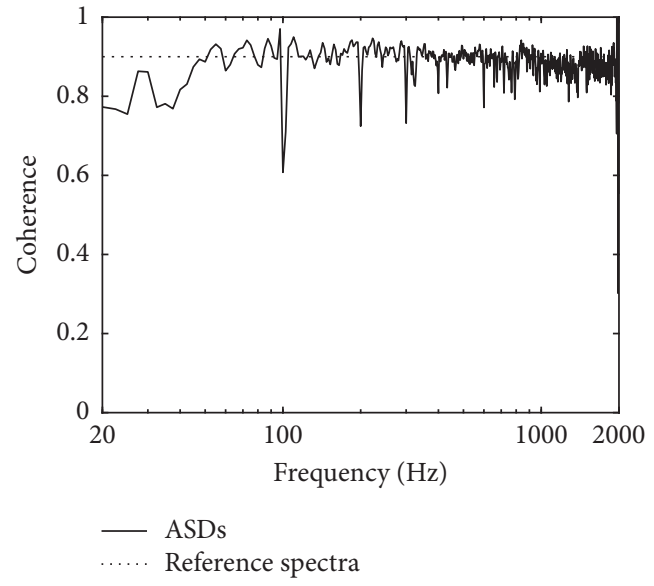

(d)

FIGURE 23: Response spectral density of the two points on the vibration table.

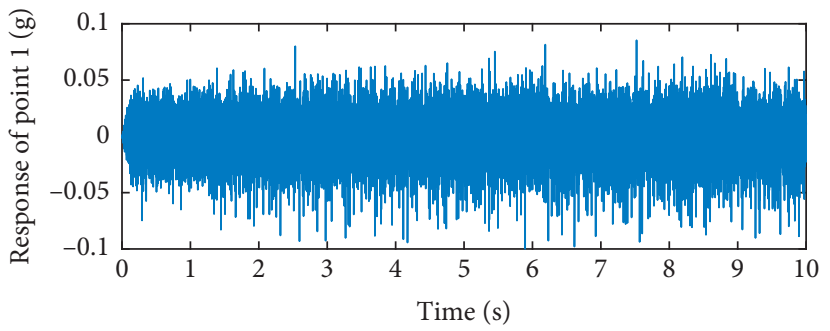

(a)

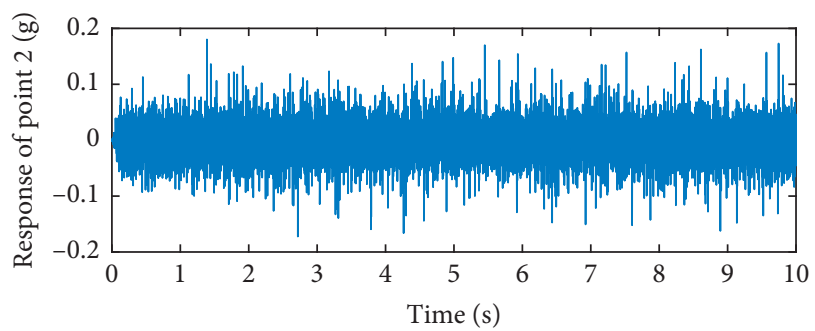

(b)

FIgURE 24: Response signals of the two points on the vibration table.

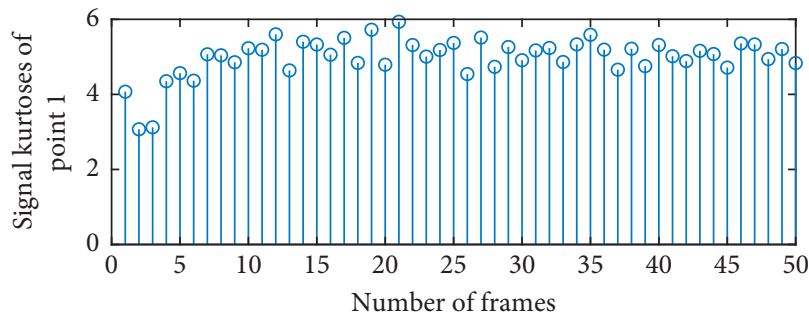

(a)

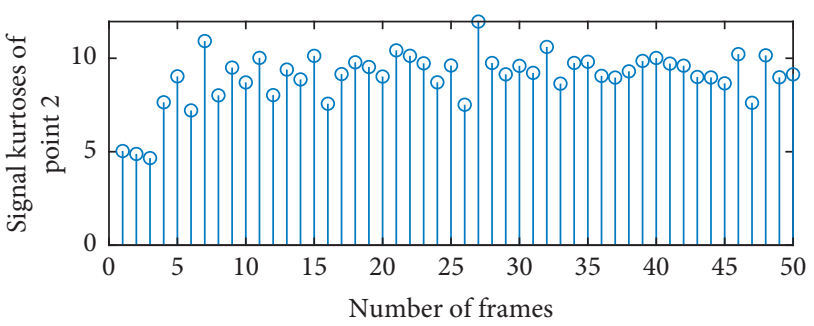

(b)

FIgURE 25: Kurtoses of the initial 50 frames of the response signals.

respectively. The parameters $b$ and $c$ are 300 and 0.6 , respectively.

Figure 22 shows the input voltage signals of the initial $10 \mathrm{~s}$. The input signals are obviously super-Gaussian. SuperGaussian inputs have much more high-excursion peaks than Gaussian or sub-Gaussian inputs, which makes the input voltage tend to exceed the voltage tolerances of the vibration table. Therefore, the WOA operation on the input voltage signals is absolutely necessary. The tolerated input-output voltage of the NI instrument is only 5; this limitation restricts us from conducting the super-Gaussian test with high kurtoses. To avoid this problem, we adopt relatively low reference spectra in this test, as seen in Figure 23. The horizontal portion of the reference spectra is only $10^{-7} \mathrm{~g}^{2} /$ $\mathrm{Hz}$. The processed input voltage signals, response signals, and the corresponding kurtoses are shown in Figures 22-25, respectively. The mean values of the kurtoses in Figure 25 are 4.98 and 9.01, almost equal to the preset reference kurtoses. Figure 23 demonstrates the power spectral densities of the response signals. The cross-power spectral densities, defined with the phase and coherence, are not disturbed by the windowed ZMNL transformation.

Providing a validation with kurtoses ranging from 4 to 40, such as in Figures 10 or 19, presents some difficulty. As 


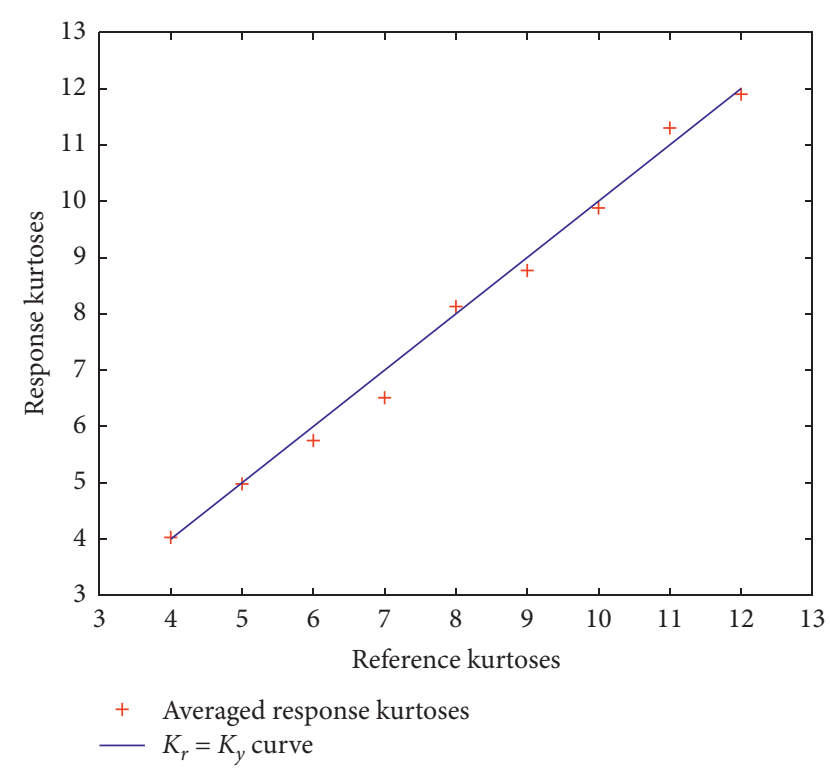

FIGURE 26: Numerical relation between the reference kurtoses and the response kurtoses: $K_{r}=K_{y}$ curve (solid line). Averaged kurtoses of the response acquired from the experiment (plus signs).

mentioned above, the hardware cannot accommodate inputs with large amplitudes when the reference kurtoses are too large. Here, a similar result with a shorter kurtosis range is given in Figure 26. In the interval from 4 to 12, the response kurtoses generally fit the $K_{r}=K_{y}$ curve.

\section{Conclusion}

In this paper, the state-space method is employed to deduce how response kurtoses are influenced by the WOA operation on the input voltage signals. The derivation in Section 4 and the numerical simulation in Section 4.3 verify that the WOA operation on the input signals is equivalent to the same operation on the corresponding response signals. Based on this finding, the reason why the WOA operation causes a kurtosis loss is studied. Then, the window operation is proposed to be added into the ZMNL transformation. With this windowed ZMNL method, high-excursion peaks of super-Gaussian signals can be centred in the middle position of every frame, and kurtoses can be invariable and even increased. A discussion about the parameter settings for the windowed ZMNL method is also presented. A singleinput single-output numerical simulation and a two-input two-output shaker table test validate the effectiveness of the proposed method.

\section{Data Availability}

The data used to support the findings of this study are available from the corresponding author upon request.

\section{Conflicts of Interest}

The authors declare no conflicts of interest.

\section{Acknowledgments}

This work was financially supported by China Postdoctoral Science Foundation (2019M651827), Jiangsu Planned Projects for Postdoctoral Research Funds (2018K042B), the Natural Science Foundation of the Jiangsu Higher Education Institutions of China (20KJD130001), and the Science Foundation of Nanjing Vocational University of Industry Technology (YK19-03-01, YK19-03-04).

\section{References}

[1] A. V. Oppenheim and R. W. Schafer, Discrete-Time Signal Processing, Prentice-Hall, Upper Saddle River, NJ, USA, 2nd edition, 1989.

[2] D. O. Smallwood and T. L. Paez, "A frequency domain method for the generation of partially coherent normal stationary time domain signals," Shock and Vibration, vol. 1, no. 1, pp. 45-53, 1993.

[3] D. O. Smallwood, "Generation of stationary non-Gaussian time histories with a specified cross-spectral density," Shock and Vibration, vol. 4, no. 5-6, pp. 361-377, 1997.

[4] A. Steinwolf, "True-random mode simulation of nonGaussian vibrations with high kurtosis values," in Proceedings of the Institute of Environmental Sciences and Technology, pp. 148-155, Mt Prospect, IL, USA, 2000.

[5] D. González and R. López-Valcarce, "Maximally stationary window design for overlap-add based random vibration synthesis," Mechanical Systems and Signal Processing, vol. 122, pp. 642-657, 2019.

[6] A. Steinwolf, "Random vibration testing with kurtosis control by IFFT phase manipulation," Mechanical Systems and Signal Processing, vol. 28, pp. 561-573, 2012.

[7] F. Cianetti, M. Palmieri, C. Braccesi, and G. Morettini, "Correction formula approach to evaluate fatigue damage induced by non-Gaussian stress state," Procedia Structural Integrity, vol. 8, pp. 390-398, 2018.

[8] D. Benasciutti and R. Tovo, "Frequency-based analysis of random fatigue loads: models, hypotheses, reality," Materialwissenschaft und Werkstoffechnik, vol. 49, no. 3, pp. 345367, 2018.

[9] F. Kihm, N. S. Ferguson, and J. Antoni, "Fatigue life from kurtosis controlled excitations," Procedia Engineering, vol. 133, pp. 698-713, 2015.

[10] V. Rouillard and M. A. Sek, "Synthesizing nonstationary, nonGaussian random vibrations," Packaging Technology and Science, vol. 23, no. 8, pp. 423-439, 2010.

[11] R. Zheng, H. Chen, D. Vandepitte, and Z. Luo, "Multi-exciter stationary non-Gaussian random vibration test with time domain randomization," Mechanical Systems and Signal Processing, vol. 122, pp. 103-116, 2019.

[12] J. Yu, X. Chen, and J. Y. Tao, "Study on the generation of super-Gaussian and true random drive signals using time domain randomization," Journal of Vibration Engineering, vol. 18, pp. 491-494, 2005.

[13] Y. Song, D. Wu, W. Deng et al., "MPPCEDE: multipopulation parallel co-evolutionary differential evolution for parameter optimization," Energy Conversion and Management, vol. 228, Article ID 113661, 2021.

[14] X. Cai, H. Zhao, S. Shang et al., "An improved quantuminspired cooperative co-evolution algorithm with mulistrategy and its application," Expert Systems with Applications, vol. 171, Article ID 114629, 2021. 
[15] J. S. Bendat and G. A. Piersol, Random Data Analysis and Measurement Procedures, IOP Publishing, Bristol, UK, 4th edition, 2000.

[16] S. Cui, E. Zheng, and M. Kang, "A time-domain procedure for non-Gaussian stationary environmental testing using zeromemory nonlinear transformation," Journal of Vibration and Control, vol. 26, no. 15-16, pp. 1197-1213, 2020.

[17] Z. Zhang and K. T. Chong, "Second order hold and Taylor series based discretization of SISO input time-delay systems," Journal of Mechanical Science and Technology, vol. 23, no. 1, pp. 136-149, 2009.

[18] Y. Ding, S. S. Law, B. Wu et al., "Average acceleration discrete algorithm for force identification in state space," Engineering Structures, vol. 56, pp. 1880-1892, 2013.

[19] S. Cui, H.-h. Chen, and X.-d. He, "Time-domain approach for multi-exciter random environment test," Journal of Sound and Vibration, vol. 398, pp. 52-69, 2017.

[20] G. A. D. o. PLA, Laboratory Environmental Test Methods for Military Materiel. GJB150A, Army Publishing, Fort Belvoir, VA, USA, 2009.

[21] K. D. Hsueh and R. P. Hamernik, "A generalized approach to random noise synthesis," The Journal of the Acoustical Society of America, vol. 85, no. S1, p. S40, 1989.

[22] J. Zhang, B. Cornelis, B. Peeters, K. Janssens, and P. Guillaume, "A new practical and intuitive method for kurtosis control in random vibration testing," in Proceedings of the ISMA, Leuven, Belgium, September 2016. 